\title{
Co-Occurrence of Colistin and Meropenem Resistance Determinants in a Stenotrophomonas Strain Isolated from Sewage Water
}

\author{
Jun Li, ${ }^{1,2,{ }^{\star}}$ Shuyan Liu, ${ }^{3, *}$ Jiafang Fu, ${ }^{4}$ Jianhua Yin, ${ }^{2}$ Jia Zhao, ${ }^{1}$ Chuanqing Zhong, ${ }^{1}$ and Guangxiang $\mathrm{CaO}^{4}$
}

Development of antibiotic resistance can be achieved either by mutation or by acquiring a resistance gene from foreign sources, with some resistance genes likely originating in microbial populations to counteract antibiotics present in natural ecosystems. In this study, we describe the first report of a strain of nonclinical multidrugresistant Stenotrophomonas sp. strain G4 with high-level resistance to colistin and meropenem, phylogenetically distinct from well-studied multiple drug-resistant species of Stenotrophomonas maltophilia. As the high-level colistin resistance of this strain was of great concern, the genome of this strain was completely sequenced. Only one chromosome was identified, and no plasmids were found. Chromosomal gene variants and other potential genetic determinants conferring resistance to colistin and meropenem were comparatively analyzed, and results showed that strain G4 harbored two putative colistin resistance determinants (named $m c r-5.3$ and $m c r-8.2$ ) and four extended-spectrum $\beta$-lactamase genes. In addition, 12 genes potentially encoding seven different types of efflux pumps were identified, which may have a major role in acquisition/transfer of colistin resistance. Our discovery of multiple antibiotic resistance determinants in this environmental strain extensively expands our understanding of the extent of dissemination of colistin and meropenem resistance.

Keywords: Stenotrophomonas, colistin, meropenem, antibiotic resistance, gene transfer

$\mathbf{T}$ HE INAPPROPRIATE USE of antibiotics is a major factor in the rapid development of drug resistance and in therapeutic failure. Therefore, it is essential to consider, and screen for, the presence of multidrug-resistant (MDR) microbes before the formulation of appropriate antibiotic-based therapies. In general, carbapenems, which are $\beta$-lactam antibiotics, are used as a last line of defense against severe infections with MDR Enterobacteriaceae, and colistin (polymyxin E), a cationic antimicrobial polypeptide, is clinically regarded as the final defense line for treating lethal challenges with carbapenem-resistant pathogens. The coexistence of colistin resistance and extended-spectrum $\beta$-lactamase (ESBL) within individual variants of bacterial species isolated from humans, animals, and the environment is a strong reminder of the need to employ new approaches for combating MDR microbes. ${ }^{1-4}$
Unfortunately, the clinical use of carbapenems and colistin has been significantly challenged by the occurrence of the mobilized colistin resistance determinant (MCR-1) in clinical carbapenem-resistant isolates that produce New Delhi metallo- $\beta$-lactamase 1 (NDM-1) ${ }^{5}$ or its variant NDM-5. ${ }^{6}$ In addition to the intrinsic determinants of colistin resistance (e.g., PmrAB and PhoPQ mutations), transferable determinants, such as MCR-1, encoding phosphoethanolamine (PEA)-lipid A transferases have been discovered. ${ }^{2,7,8}$ The mechanism of action of MCR involves the covalent addition of PEA to the $4^{\prime}$-phosphate position of lipopolysaccharide lipid A moieties through a "ping-pong" trade-off. ${ }^{7-10}$ The increasing incidence of MCR-like determinants, including MCR-1, MCR-2, MCR-3, MCR-4, MCR-5, MCR-6, MCR-7, and the recently reported MCR-8, which are found in bacteria in patients, animals, and various environments, challenges the

\footnotetext{
${ }^{1}$ School of Municipal and Environmental Engineering, Shandong Jianzhu University, Jinan, China.

${ }^{2}$ College of Biotechnology and Bioengineering, Zhejiang University of Technology, Hangzhou, China.

${ }^{3}$ Zhejiang Provincial People's Hospital, Hangzhou, China.

${ }^{4}$ Shandong Medicinal Biotechnology Center, Shandong Academy of Medical Sciences, Jinan, China.

*These authors contributed equally to this study.
}

(C) Jun Li et al., 2019; Published by Mary Ann Liebert, Inc. This Open Access article is distributed under the terms of the Creative Commons Attribution Noncommercial License (http://creativecommons.org/licenses/by-nc/4.0/) which permits any noncommercial use, distribution, and reproduction in any medium, provided the original author(s) and the source are cited. 
renewed interest in colistin for clinical therapies. In particular, MCR-1 and MCR-2 are commonly carried by plasmids, and $>10$ genetic variants of MCR-1/2 have been detected from these transferable reservoirs, suggesting the ongoing spread and dissemination of MCR-1/2. ${ }^{9}$ The transferability of MCRlike polymyxin resistance is of social concern, as MCR-like mechanisms pose a serious threat to public health and the safety of the food chain. Furthermore, although rare, MCR has already been found to coexist with both $\mathrm{ESBL}^{11}$ and NDM- $1^{12}$ (or its variants NDM- $5^{13}$ and NDM- $9^{14}$ ).

In this study, we report on a nonclinical strain of MDR Stenotrophomonas sp., named G4, coharboring MCR- and ESBL-genetic determinants, which was isolated from sewage water of a poultry farm in Jinan, China, and likely developed its resistance pattern from antibiotic exposure and overuse in an agricultural setting. The sewage water was gradiently diluted with sterilized water, and then plated onto LB agar supplemented with $8 \mu \mathrm{g} / \mathrm{mL}$ colistin and $8 \mu \mathrm{g} / \mathrm{mL}$ meropenem, and incubated at $30^{\circ} \mathrm{C}$ for 24 hour to obtain single colonies; then one of the single colonies was picked out and streaked on the above LB agar to obtain a pure culture. After purification, a single colony (representing a pure culture) was named as strain G4. The G4 strain was cultured with LB supplemented with $8 \mu \mathrm{g} / \mathrm{mL}$ colistin and $8 \mu \mathrm{g} / \mathrm{mL}$ meropenem, and then the cells were harvested and the total DNA was isolated. The primer pairs $27 \mathrm{~F}\left(5^{\prime}-\right.$ AGAGTTTGATCCTGGCTCAG-3') and 1492R (5'-GGT TACCTTGTTACGACTT-3') were used to amplify the partial fragment of 16S rDNA. Strain G4 was identified as Stenotrophomonas sp. based on 16S rDNA sequence analysis (GenBank Accession number: MK418754). A background survey found that amoxicillin and colistin had been regularly used in this livestock farm for poultry to prevent infection. To determine the minimal inhibitory concentrations (MICs) of strain G4 for different antibiotics, including ampicillin, cefixime, ciprofloxacin, florfenicol, meropenem, colistin, and tetracycline, the broth microdilution method was used, as described by the Clinical Laboratory Standard Institute (CLSI) guidelines. ${ }^{15}$ Results showed that strain G4 has a striking resistance to the antibiotics tested (Table 1). Compared with the published MICs for colistin-susceptible Klebsiella pneumoniae, Escherichia coli, and Stenotrophomonas sp. strains, ${ }^{2,3,16-18}$ the MICs of colistin and meropenem for strain G4 were higher.

To determine the genetic basis for the multidrug-resistance phenotype of this strain, whole-genome sequencing was performed using the Illumina NextSeq platform and the PacBio RS II platform. The resultant contigs were assembled by SOAPdenovo (soap.genomics.org.cn) and Velvet (ebi.sv .uk/ zerbino/velvet) programs, generating a total genome sequence of $4,477,485 \mathrm{bp}$ with an average GC content of
$66.83 \%$. The features of the complete genome sequence are shown in Supplementary Table S1. Whole genome alignment by BLASTp-based searches and a core genomic phylogeny established with CVTree ${ }^{19}$ both grouped strain G4 with Stenotrophomonas species (Figs. 1 and 2), and strain G4 phylogenetic clade was close to Stenotrophomonas rhizophila and Stenotrophomonas maltophilia. S. rhizophila DSM14405 can facilitate plant growth and alleviate adversity. ${ }^{20}$ Moreover, S. maltophilia pertaining to Stenotrophomonas species is the only phylogenetic clade known to cause human disease and prompt broad antibiotic resistance, ${ }^{17,21,22}$ and a recent study has described the presence of specific subgroups of $S$. maltophilia strains adapted to the human host resulting from their genomic diversity and variation. ${ }^{22}$ As shown in Fig. 1, strain G4 was more closely related to $S$. rhizophila rather than S. maltophilia and Xanthomonas species, based on whole genomic level phylogeny with $S$. rhizophila DSM14405, four $S$. maltophilia and a variety of closely related Xanthomonas fully sequenced genomes in GenBank. However, the more accurate species assignment of strain G4 needs further clarified with the Biolog examination ${ }^{23}$ and more fully sequenced Stenotrophomonas strains.

As strain G4 has notably high levels of colistin and $\beta$ lactam resistance, we conducted comparative genomics to identify potential MCR- and ESBL-encoding genes, using the circular genome of strain G4 and closely related Stenotrophomonas spp. (Fig. 2). In addition, four genes coding for $\beta$-lactamases and two $\mathrm{mcr}$-like genes were predicted (Table 2) using the ARG-ANNOT and VRprofile programs. ${ }^{24,25} \mathrm{We}$ renamed the $m c r-5$-like gene, which contains mutations compared with the prototype $m c r-5$, as $m c r-5.3$, whereas the $m c r-8$-like gene was designated $m c r-8.2$ (Fig. 3 and Supplementary Fig. S1). The genomic contexts of the MCRencoding genes were also assessed to investigate their transmission potentials and spread risk (Fig. 4). The mcr-pap2 unit was observed to be widely disseminated in species closely related to strain G4 (Fig. 4). However, no IS element, such as ISApl1, which is usually contained by a $m c r-1 / 2$ cassette [ISApl1-mcr-1-orf-ISApl1, presumptive Tn6330], ${ }^{10}$ was found in the strain G4 genome. Therefore, strain G4 may comprise a reservoir of $m c r$ genes to facilitate their spread and persistence. Similarly, four genes coding for ESBLs and which represent intrinsic antibiotic resistance capabilities were identified, highlighting the foreign and ancestral origins of ESBL- and MCR-related antibiotic resistance determinants. Group 3c $\beta$-lactamase $\left(\right.$ bla $\left._{\mathrm{L} 1}\right)$ and Group 2e $\beta$-lactamase $\left(b_{\mathrm{L} 2}\right)$, responsible for the typical $\beta$-lactam resistance properties of $S$. maltophilia, were discovered in strain G4 (Table 2). In $S$. maltophilia, the bla $_{\mathrm{L} 1-\text { encoded broad- }}$ spectrum metallo- $\beta$-lactamase has been found to hydrolyze

Table 1. Minimal Inhibitory Concentrations of Antibiotics for Strain G4 AND ESCHERICHIA COLI CONJUGANTS

\begin{tabular}{lcccccr}
\hline Strain & Ampicillin* & Cefixime & Colistin & Florfenicol & Meropenem & Tetracycline \\
\hline G4 & $>256$ & $>256$ & 160 & 32 & 144 & 40 \\
25DN** & 8 & $<2$ & $<2$ & $<2$ & $<2$ & 4 \\
25DN-POL & 12 & $<2$ & 72 & $<2$ & $<2$ & 4 \\
\hline
\end{tabular}

*Concentration units for the antibiotics tested: $\mu \mathrm{g} / \mathrm{mL}$

$* * 25 \mathrm{DN}$, sodium azide-resistant $E$. coli strain; 25DN-POL conjugant derived after colistin selection, with $25 \mathrm{DN}$ as recipient strain and G4 as donor strain. 


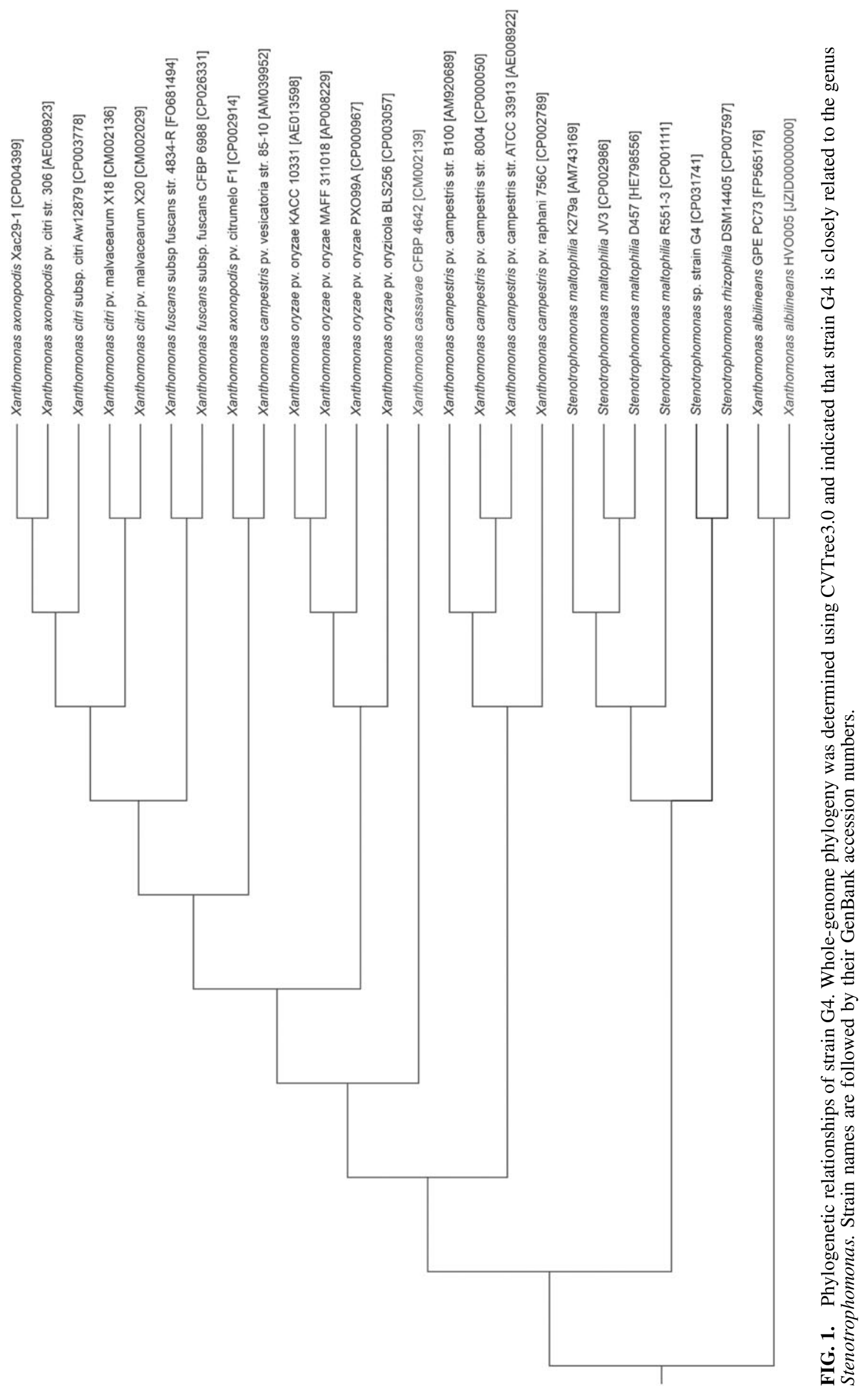




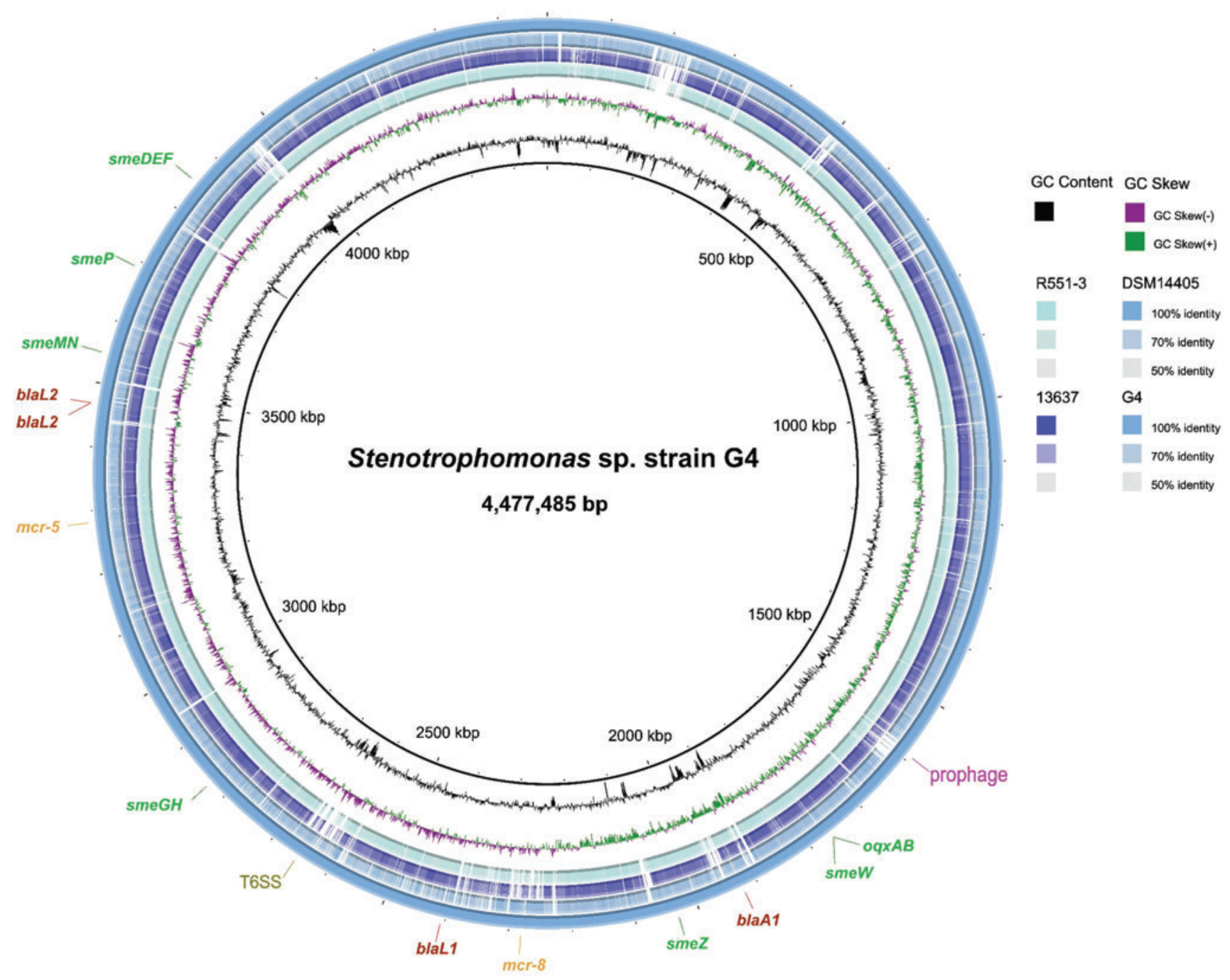

FIG. 2. Circular schematics of the strain G4 genome and its BLASTn-based comparison with closely related bacterial genomes. Genomes of Stenotrophomonas strains DSM14405, R551-3, and 13637 were chosen for reference. Color images are available online.

Table 2. Identified Antibiotic Resistance Genetic Determinants and Their Locus Tags in the Strain G4 Genome

Efflux pumps [gene (locus tag*)]

\begin{tabular}{lcccccc}
\hline smeDEF & smeMN & smeGH & smeP & $m d t A$ & smeZ & oqxAB \\
\hline$(3512-4)$ & $(3246-7)$ & $(2598-9)$ & $(3369)$ & $(3370)$ & $(1894)$ & $(1655-6)$ \\
\hline
\end{tabular}

$\beta$-lactams resistance [gene (locus tag)]

\begin{tabular}{llll}
\hline$b l a_{\mathrm{L} 1}$ & $b l a_{\mathrm{L} 2}$ & $b l a_{\mathrm{L} 2}$ & $b l a_{\mathrm{A} 1}$ \\
\hline$(2219)$ & $(3176)$ & $(3177)$ & $(1799)$ \\
\hline
\end{tabular}

Colistin resistance [gene (locus tag)]

\begin{tabular}{ll}
\hline$m c r-5$ & $m c r-8$ \\
\hline$(3021)$ & $(2100)$ \\
\hline
\end{tabular}

*Gene locus tags: ORF_No. for the abbreviation of G4GL00(ORF_No.), such as 3512-4 indicating ORFs from G4GL003512 to G4GL003514. 


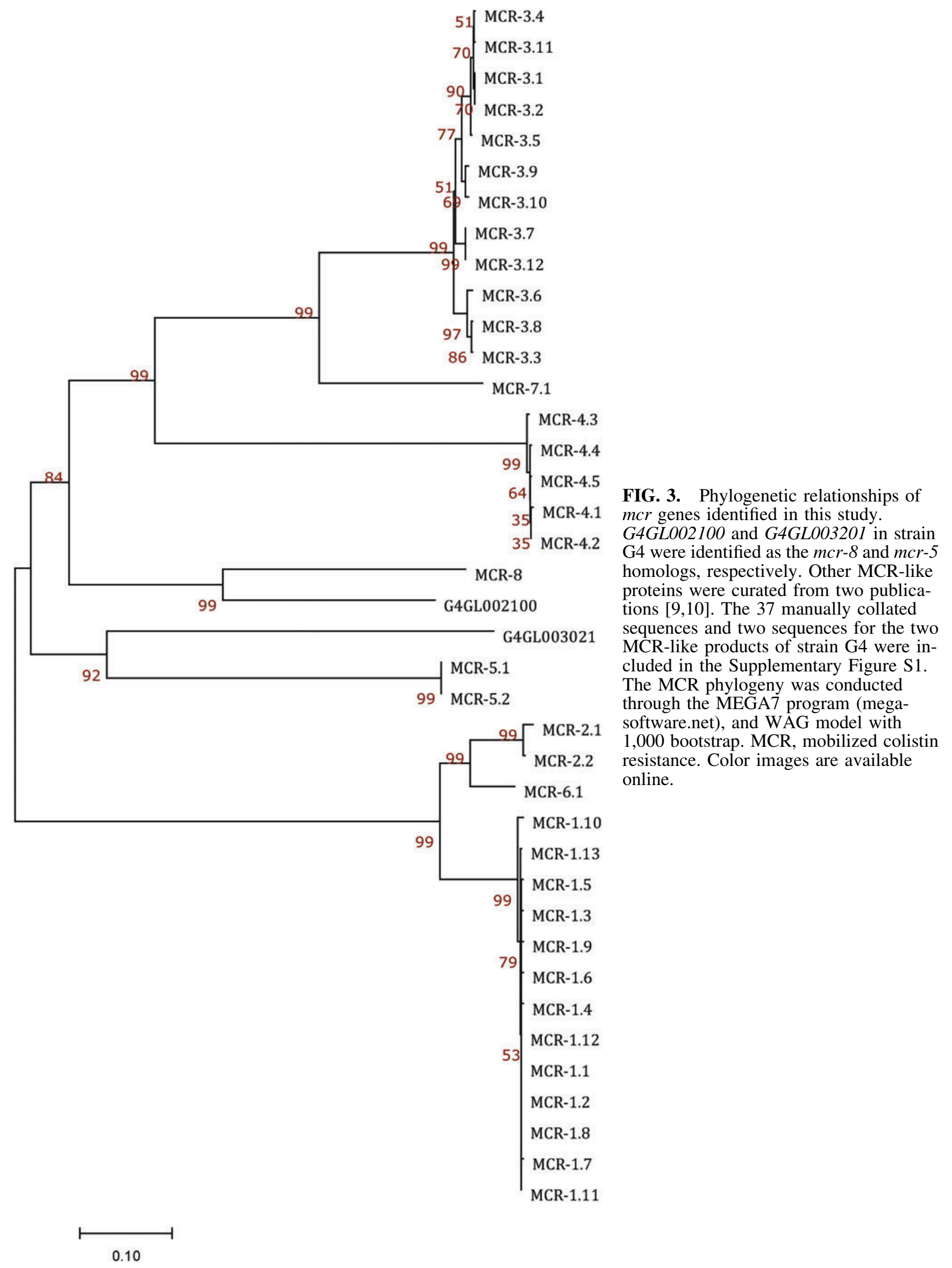




\section{A}
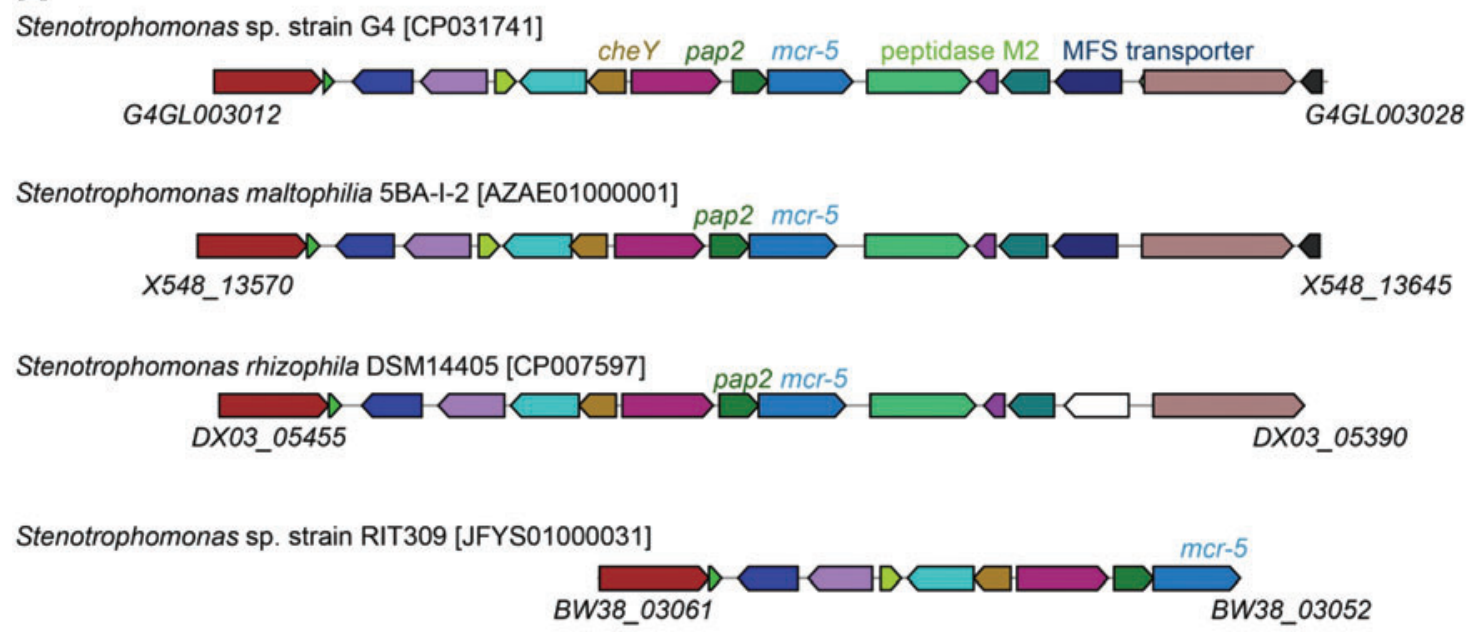

B
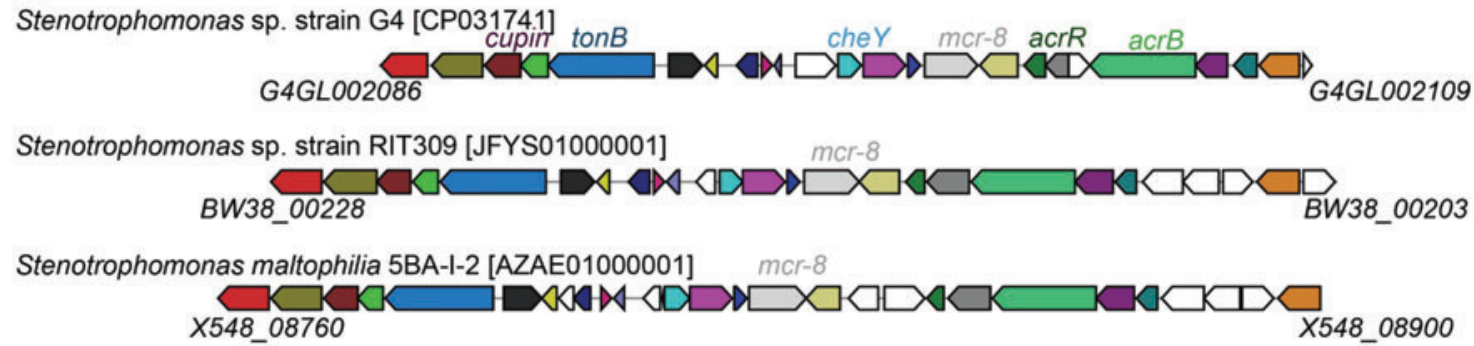

Stenotrophomonas rhizophila DSM14405 [CP007597]

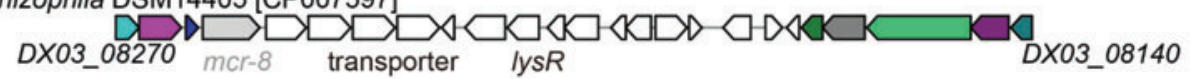

FIG. 4. Genomic contexts of $m c r$-like genes in the strain G4 genome and closely related strains. (A) $m c r$-5-like genes are shown in light blue; (B) mcr-8-like genes are shown in light gray. Strain names are followed by their GenBank accession numbers, and homologous genes are indicated by matching colors. Color images are available online.

carbapenems. ${ }^{26}$ In summary, the aforementioned $\beta$-lactamase antimicrobial resistance (AMR) determinants in strain $\mathrm{G} 4$ are likely intrinsic progenitors, which may have been widely disseminated to other species.

RND- and AcrAB-type efflux pumps that increase resistance to antimicrobial agents have been recently reported and are also involved in colistin and $\beta$-lactam resistance. ${ }^{17,27,28}$ The strain G4 chromosome encodes seven efflux pumps, including SmeDEF, SmeMN, SmeGH, SmeP, SmeW, SmeZ, and OqxAB (Table 2). In Stenotrophomonas species phylogenetically related to strain G4, the overproduction of efflux pumps, such as SmeDEF, confers highlevel antibiotic resistance; moreover, antibiotic extrusion and colonization are both likely related to SmeDEF. ${ }^{17}$ The expression and activity of efflux pumps are usually inhibited, but SmeDEF expression could be induced by plant-produced flavonoids and, therefore, impact antibiotic resistance. ${ }^{17}$ Since SmeDEF expression can be triggered by plant-produced flavonoids in Stenotrophomonas, we hypothesized that this efflux pump might have a role in antibiotic resistance and the colonization of plants by strain G4.

The strain G4 chromosome contains an intact prophage (T6SS) as well as one type VI secretion system (Fig. 5). Prophages have been previously determined to contribute to host specificity of pathogens and growth fitness. ${ }^{25,29}$ Of note, a variable region of strain G4 was comparatively identified in the prophage, and this unstable region and its vicinity are proposed to correlate with strain G4 environmental adaptability (Fig. 5A). The genetic arrangement of the T6SS of strain G4 is almost identical to that of its counterparts in other closely related strains and to that of the type i4b T6SS in Xanthomonas oryzae ${ }^{30}$ (Fig. 5B). Type i4b T6SSs have been experimentally verified to provide virulence traits and alternative adaptabilities in other strains. $^{31-33}$ Given that the activation of T6SS renders Acinetobacter baumannii more susceptible to antibiotics, ${ }^{34,35}$ it will be important to elucidate the role of T6SS in the antibiotic resistance of the MDR strain G4.

To determine if the antibiotic resistance genes could be transferred from strain G4 to other bacteria by horizontal gene transfer, the sodium azide-resistant $E$. coli $25 \mathrm{DN}$ strain (derived from $E$. coli 25922) was used as the recipient strain for examining conjugal transfer of resistance. Colistin and meropenem were used to select the resistant conjugants, as described previously. ${ }^{36}$ For example, after mixing the donor strain G4 and recipient strain 25DN, the mixture was spread on LB agar medium with X-Gluc (5-bromo-4-chloro-3indolyl- $\beta$-D-glucuronic acid), sodium azide, and $8 \mu \mathrm{g} / \mathrm{mL}$ colistin to screen for colistin-resistant conjugants. As strain G4 was inhibited by sodium azide, and 25DN was inhibited 

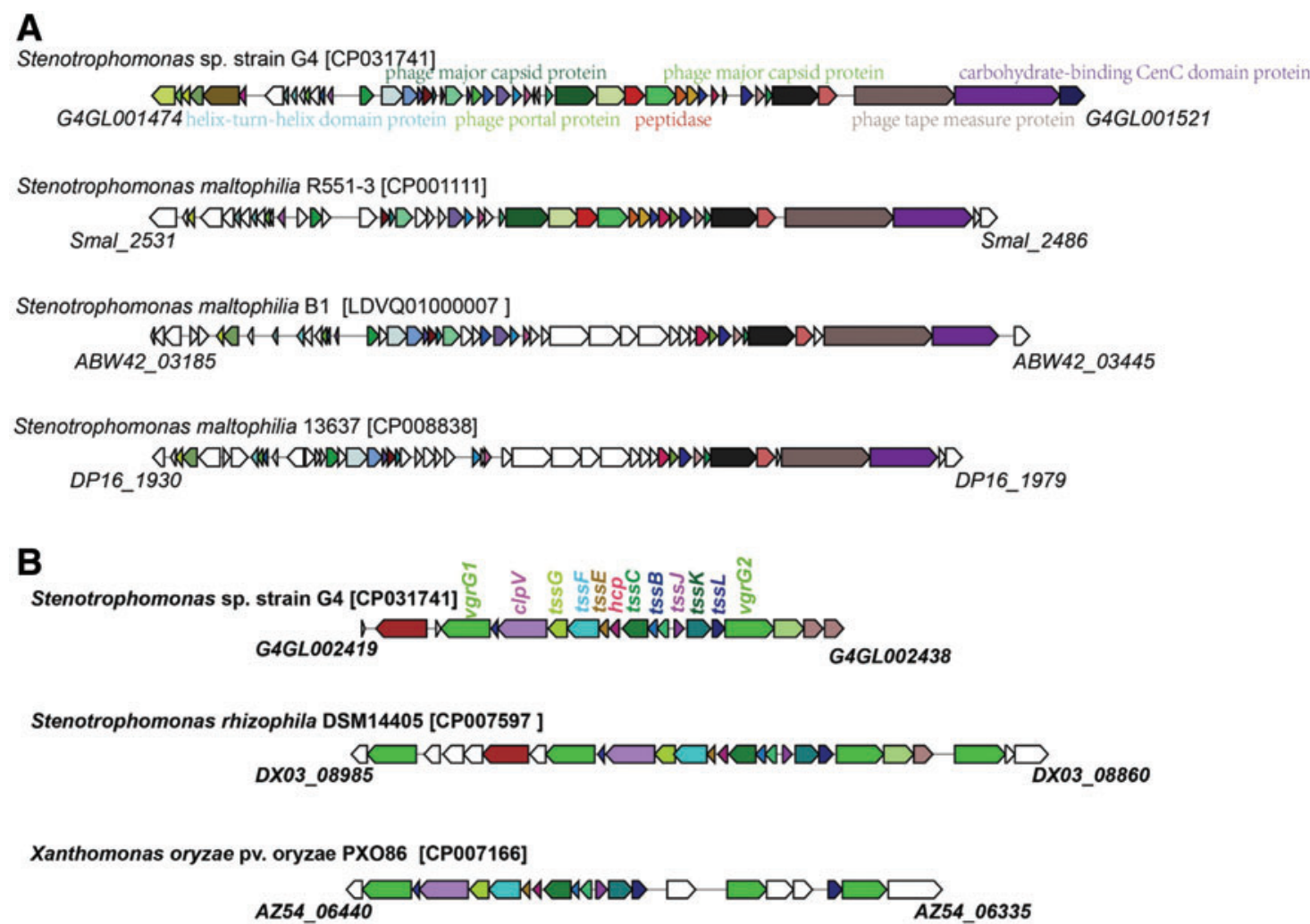

Xanthomonas oryzae pv. oryzae MAFF 311018 [AP008229]

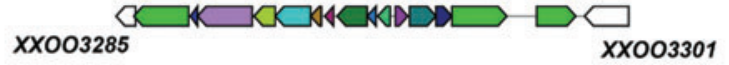

FIG. 5. Synteny analyses of the gene clusters coding for (A) an intact prophage and (B) a type VI secretion system. Strain names are followed by their GenBank accession numbers, and homologous genes are indicated by matching colors. Color images are available online.

by $8 \mu \mathrm{g} / \mathrm{mL}$ colistin, only the conjugants could survive on the selective medium and degrade X-Gluc to blue compounds. The conjugal transfer efficiency between $\mathrm{G} 4$ and $25 \mathrm{DN}$ was about $1.33 \times 10^{-6} / \mathrm{donor}$ using $8 \mu \mathrm{g} / \mathrm{ml}$ colistin as the selective pressure. One of these conjugants was isolated and named 25DN-POL. The MIC of colistin for 25DN-POL was $72 \mu \mathrm{g} / \mathrm{mL}$ (Table 1), considerably higher than for the recipient strain $25 \mathrm{DN}$, whereas the MICs for other antibiotics were similar to those for $25 \mathrm{DN}$, except for ampicillin. Instead, no conjugant was found using $8 \mu \mathrm{g} / \mathrm{mL}$ meropenem as the selective pressure. These results indicated that the colistin resistance of strain $\mathrm{G} 4$ can be partially transferred to E. coli by horizontal gene transfer. As strain G4 harbors a variety of mobile elements near smeDEF, smeMN, smeP, smeZ, and $m d t A$ genes, and no mobile elements near $m c r-5.3$ and $m c r-8.2$ genes (Supplementary Table S2), the mechanism of acquisition/transfer of colistin resistance in the G4 strain may be related to efflux pumps.

Antibiotic resistance genes and their transfer are an urgent risk to human health. ${ }^{37,38}$ It has been frequently reported that $m c r$-like genes could cotransfer with other antibiotic resistance genetic determinants, such as NDM-1 gene and/or its derivates (NDM-5 and NDM-9)., ${ }^{5,39}$ This genetic colocalization of a variety of AMR genes raises the possibility that bacteria could acquire resistance to both colistin and carbapenem, two categories of antibiotics that are both considered the last resorts against MDR bacteria. It is likely that Enterobacteriaceae could predominantly account for the $m c r$-like genes in commensal and gut microbe. ${ }^{9,10,40}$ However, our discovery of a Stenotrophomonas strain possessing two MCR-like determinants and several ESBL genes implies that the sources and distribution of AMR genes may be substantially complex. Presently, the emergence and transmission of MCR and ESBL pose great risks to public health through the food chain and environmental exposure. Based on this scenario, the proposed "one health" approach ${ }^{10}$ might benefit the effective control of worldwide AMR problems and assist combating the stringent challenges from MDR superbugs, which requires the systemic coordination of three sectors: environment, animals, and humans.

\section{Data Availability}

Complete sequence of the strain G4 genome was deposited into GenBank under accession No. CP031741, and 16S rRNA sequence of the strain G4 was submitted to GenBank assigned with accession No. MK418754.

\section{Acknowledgments}

We thank Dr. Susan T. Howard for critical reading of the article. This study was supported by the National Natural Science Foundation of China (31800118 and 31500094), 
China Postdoctoral Science Foundation (2017M621965), and the Innovation Project of Shandong Academy of Medical Sciences.

\section{Author Contributions}

J.L. and G.C. designed and supervised this project; S.L., J.Z., and J.F. performed experiments; J.L., J.Y., C.Z., and G.C. analyzed the data; J.L., C.Z., and G.C. drafted this article.

\section{Disclosure Statement}

Authors declared no potential conflict of interest.

\section{Supplementary Material}

Supplementary Figure S1

Supplementary Table S1

Supplementary Table S2

\section{References}

1. Gao, R., Q. Wang, P. Li, Z. Li, and Y. Feng. 2016. Genome sequence and characteristics of plasmid $\mathrm{pWH} 12$, a variant of the mcr-1-harbouring plasmid pHNSHP45, from the multi-drug resistant E. coli. Virulence. 7:732-735.

2. Wang, Q., J. Sun, J. Li, Y. Ding, X.P. Li, J. Lin, B. Hassan, and Y. Feng. 2017. Expanding landscapes of the diversified $m c r-1$-bearing plasmid reservoirs. Microbiome. 5:70.

3. Wang, X., Y. Wang, Y.Zhou, J. Li, W. Yin, S. Wang, S. Zhang, J. Shen, Z. Shen, and Y. Wang. 2018. Emergence of a novel mobile colistin resistance gene, $m c r-8$, in NDM-producing Klebsiella pneumoniae. Emerg. Microbes. Infect. 7:122.

4. Teo, J.W.P., M. Kalisvar, I. Venkatachalam, O.T. Ng, R.T.P. Lin, and S. Octavia. 2018. $m c r-3$ and $m c r-4$ variants in carbapenemase-producing clinical Enterobacteriaceae do not confer phenotypic polymyxin resistance. J. Clin. Microbiol. 56:e01562-17.

5. Ranjan, A., S. Shaik, A. Mondal, N. Nandanwar, A. Hussain, T. Semmler, N. Kumar, S.K. Tiwari, S. Jadhav, L.H. Wieler, and N. Ahmed. 2016. Molecular epidemiology and genome dynamics of New Delhi metallo-beta-lactamaseproducing extraintestinal pathogenic Escherichia coli strains from India. Antimicrob. Agents. Chemother. 60: 6795-6805.

6. Hornsey, M., L. Phee, and D.W. Wareham. 2011. A novel variant, NDM-5, of the New Delhi metallo-beta-lactamase in a multidrug-resistant Escherichia coli ST648 isolate recovered from a patient in the United Kingdom. Antimicrob. Agents. Chemother. 55:5952-5954.

7. Sun, J., Y. Xu, R. Gao, J. Lin, W. Wei, S. Srinivas, D. Li, R.S. Yang, X.P. Li, X.P. Liao, Y.H. Liu, and Y. Feng. 2017. Deciphering MCR-2 colistin resistance. MBio. 8: e00625-17.

8. Wei, W., S. Srinivas, J. Lin, Z. Tang, S. Wang, S. Ullah, V.G. Kota, and Y. Feng. 2018. Defining ICR-Mo, an intrinsic colistin resistance determinant from Moraxella osloensis. PLoS. Genet. 14:e1007389.

9. Partridge, S.R., V. Di Pilato, Y. Doi, M. Feldgarden, D.H. Haft, W. Klimke, S. Kumar-Singh, J.H. Liu, S. MalhotraKumar, A. Prasad, G.M. Rossolini, S. Schwarz, J. Shen, T. Walsh, Y. Wang, and B.B. Xavier. 2018. Proposal for assignment of allele numbers for mobile colistin resistance ( $m c r$ ) genes. J. Antimicrob. Chemother. 73:2625-2630.
10. Sun, J., H. Zhang, Y.H. Liu, and Y. Feng. 2018. Towards understanding MCR-like colistin resistance. Trends. Microbiol. 26:794-808.

11. Haenni, M., V. Metayer, E. Gay, and J.Y. Madec. 2016. Increasing trends in $m c r-1$ prevalence among extendedspectrum-beta-lactamase-producing Escherichia coli isolates from French calves despite decreasing exposure to colistin. Antimicrob. Agents. Chemother. 60:6433-6434.

12. Delgado-Blas, J.F., C.M. Ovejero, L. Abadia-Patino, and B. Gonzalez-Zorn. 2016. Coexistence of $m c r-1$ and $b l a_{\mathrm{NDM}-1}$ in Escherichia coli from Venezuela. Antimicrob. Agents. Chemother. 60:6356-6358.

13. Du, H., L. Chen, Y.W. Tang, and B.N. Kreiswirth. 2016. Emergence of the $m c r-1$ colistin resistance gene in carbapenem-resistant Enterobacteriaceae. Lancet. Infect. Dis. 16:287-288.

14. Yao, X., Y. Doi, L. Zeng, L. Lv, and J.H. Liu. 2016. Carbapenem-resistant and colistin-resistant Escherichia coli co-producing NDM-9 and MCR-1. Lancet. Infect. Dis. 16:288-289.

15. Behera, B., P. Mathur, A. Das, A. Kapil, B. Gupta, S. Bhoi, K. Farooque, V. Sharma, and M.C. Misra. 2010. Evaluation of susceptibility testing methods for polymyxin. Int. J. Infect. Dis. 14:e596-601.

16. Stoesser, N., A.J. Mathers, C.E. Moore, N.P. Day, and D.W. Crook. 2016. Colistin resistance gene mcr-1 and pHNSHP45 plasmid in human isolates of Escherichia coli and Klebsiella pneumoniae. Lancet. Infect. Dis. 16:285-286.

17. Ni, W., Y. Li, J. Guan, J. Zhao, J. Cui, R. Wang, and Y. Liu. 2016. Effects of efflux pump inhibitors on colistin resistance in multidrug-resistant gram-negative bacteria. Antimicrob. Agents. Chemother. 60:3215-3218.

18. Liu, Y.Y., Y. Wang, T.R. Walsh, L.X. Yi, R. Zhang, J. Spencer, Y. Doi, G. Tian, B. Dong, X. Huang, L.F. Yu, D. Gu, H. Ren, X. Chen, L. Lv, D. He, H. Zhou, Z. Liang, J.H. Liu, and J. Shen. 2016. Emergence of plasmid-mediated colistin resistance mechanism MCR-1 in animals and human beings in China: a microbiological and molecular biological study. Lancet. Infect. Dis. 16:161-168.

19. Xu, Z., and B. Hao. 2009. CVTree update: a newly designed phylogenetic study platform using composition vectors and whole genomes. Nucleic. Acids. Res. 37: W174-W178.

20. Alavi, P., M.R. Starcher, C. Zachow, H. Muller, and G. Berg. 2013. Root-microbe systems: the effect and mode of interaction of Stress Protecting Agent (SPA) Stenotrophomonas rhizophila DSM14405(T.). Front. Plant. Sci. 4:141.

21. Ryan, R.P., S. Monchy, M. Cardinale, S. Taghavi, L. Crossman, M.B. Avison, G. Berg, D. van der Lelie, and J.M. Dow. 2009. The versatility and adaptation of bacteria from the genus Stenotrophomonas. Nat. Rev. Microbiol. 7: 514-525.

22. Steinmann, J., U. Mamat, E.M. Abda, L. Kirchhoff, W.R. Streit, U.E. Schaible, S. Niemann, and T.A. Kohl. 2018. Analysis of phylogenetic variation of Stenotrophomonas maltophilia reveals human-specific branches. Front. Microbiol. 9:806.

23. Blanco, P., F. Corona, and J.L. Martinez. 2018. Biolog phenotype microarray is a tool for the identification of multidrug resistance efflux pump inducers. Antimicrob. Agents. Chemother. 62:e01263-18.

24. Gupta, S.K., B.R. Padmanabhan, S.M. Diene, R. LopezRojas, M. Kempf, L. Landraud, and J.M. Rolain. 2014. 
ARG-ANNOT, a new bioinformatic tool to discover antibiotic resistance genes in bacterial genomes. Antimicrob. Agents. Chemother. 58:212-220.

25. Li, J., C. Tai, Z. Deng, W. Zhong, Y. He, and H.Y. Ou. 2018. VRprofile: gene-cluster-detection-based profiling of virulence and antibiotic resistance traits encoded within genome sequences of pathogenic bacteria. Brief. Bioinform. 19:566-574.

26. Avison, M.B., C.S. Higgins, C.J. von Heldreich, P.M. Bennett, and T.R. Walsh. 2001. Plasmid location and molecular heterogeneity of the L1 and L2 beta-lactamase genes of Stenotrophomonas maltophilia. Antimicrob. Agents. Chemother. 45:413-419.

27. Sheng, Z.K., F. Hu, W. Wang, Q. Guo, Z. Chen, X. Xu, D. Zhu, and M. Wang. 2014. Mechanisms of tigecycline resistance among Klebsiella pneumoniae clinical isolates. Antimicrob. Agents. Chemother. 58:6982-6985.

28. Bi, D., J. Zheng, J.J. Li, Z.K. Sheng, X. Zhu, H.Y. Ou, Q. $\mathrm{Li}$, and Q. Wei. 2018. In silico typing and comparative genomic analysis of IncFIIK plasmids: insights into the evolution of replicons, plasmid backbones and resistance determinant profiles. Antimicrob. Agents. Chemother. 62: e00764-18.

29. Fan, X., A.A. Abd Alla, and J. Xie. 2016. Distribution and function of prophage phiRv1 and phiRv2 among Mycobacterium tuberculosis complex. J. Biomol. Struct. Dyn. 34:233-238.

30. Li, J., Y. Yao, H.H. Xu, L. Hao, Z. Deng, K. Rajakumar, and H.Y. Ou. 2015. SecReT6: a web-based resource for type VI secretion systems found in bacteria. Environ. Microbiol. 17:2196-2202.

31. Si, M., Y. Wang, B. Zhang, C. Zhao, Y. Kang, H. Bai, D. Wei, L. Zhu, L. Zhang, T.G. Dong, and X. Shen. 2017. The type VI secretion system engages a redox-regulated dual-functional heme transporter for zinc acquisition. Cell. Rep. 20:949-959.

32. Russell, A.B., M. LeRoux, K. Hathazi, D.M. Agnello, T. Ishikawa, P.A. Wiggins, S.N. Wai, and J.D. Mougous. 2013. Diverse type VI secretion phospholipases are functionally plastic antibacterial effectors. Nature. 496:508-512.

33. Verster, A.J., B.D. Ross, M.C. Radey, Y. Bao, A.L. Goodman, J.D. Mougous, and E. Borenstein. 2017. The landscape of type VI secretion across human gut microbiomes reveals its role in community composition. Cell. Host. Microbe. 22:411-419 e414.

34. Weber, B.S., P.M. Ly, J.N. Irwin, S. Pukatzki, and M.F. Feldman. 2015. A multidrug resistance plasmid contains the molecular switch for type VI secretion in Acinetobacter baumannii. Proc. Natl. Acad. Sci. U. S. A. 112:9442-9447.
35. Di Venanzio, G., K.H. Moon, B.S. Weber, J. Lopez, P.M. Ly, R.F. Potter, G. Dantas, and M.F. Feldman. 2019. Multidrug-resistant plasmids repress chromosomally encoded T6SS to enable their dissemination. Proc. Natl. Acad. Sci. U. S. A. 116:1378-1383

36. Zhong, C., C. Zhang, J. Fu, W. Chen, T. Jiang, and G. Cao. 2018. Complete genome sequence of Enterobacter cloacae R11 reveals multiple genes potentially associated with highlevel polymyxin E resistance. Can. J. Microbiol. 64:87-90.

37. McArthur, A.G., N. Waglechner, F. Nizam, A. Yan, M.A. Azad, A.J. Baylay, K. Bhullar, M.J. Canova, G. De Pascale, L. Ejim, L. Kalan, A.M. King, K. Koteva, M. Morar, M.R. Mulvey, J.S. O'Brien, A.C. Pawlowski, L.J. Piddock, P. Spanogiannopoulos, A.D. Sutherland, I. Tang, P.L. Taylor, M. Thaker, W. Wang, M. Yan, T. Yu, and G.D. Wright. 2013. The comprehensive antibiotic resistance database. Antimicrob. Agents. Chemother. 57:3348-3357.

38. Partridge, S.R., and G. Tsafnat. 2018. Automated annotation of mobile antibiotic resistance in Gram-negative bacteria: the Multiple Antibiotic Resistance Annotator (MARA) and database. J. Antimicrob. Chemother. 73:883-890.

39. Hu, X., X. Xu, X. Wang, W. Xue, H. Zhou, L. Zhang, Q. Ma, R. Zhao, G. Li, P. Li, C. Zhang, Y. Shi, J. Wang, L. Jia, R. Hao, L. Wang, D. Zou, X. Liu, S. Qiu, H. Song, and Y. Sun. 2017. Diversity of New Delhi metallo-beta-lactamaseproducing bacteria in China. Int. J. Infect. Dis. 55:92-95.

40. Feng, Y. 2018. Transferability of MCR-1/2 polymyxin resistance: complex dissemination and genetic mechanism. ACS. Infect. Dis. 4:291-300.

Address correspondence to: Chuanqing Zhong, PhD

School of Municipal and Environmental Engineering Shandong Jianzhu University Jinan 250101

China

E-mail: zhongchuanqing@sdjzu.edu.cn

Guangxiang Cao, PhD

Shandong Medicinal Biotechnology Center Shandong Academy of Medical Sciences Jinan 250062

China

E-mail: caozhong0402@163.com 